\title{
Admissions for post-discharge surgical site infection at a quaternary South African public sector hospital
}

\author{
N Naidoo, ${ }^{1}$ TE Madiba, ${ }^{1}$ Y Moodley $^{2}$ \\ ${ }^{1}$ Department of Surgery, Nelson R. Mandela School of Medicine, University of KwaZulu-Natal, South Africa \\ ${ }^{2}$ Department of Anaesthetics, Nelson R. Mandela School of Medicine, University of KwaZulu-Natal, South Africa
}

Corresponding author: Dr Natasha Naidoo (drnnaidoo@icloud.com)

\begin{abstract}
Background: Reports of post-discharge admissions for surgical site infection (SSI) in African settings are lacking. This information could assist with allocating resources within hospitals, as well as developing targeted interventions aimed at reducing post-discharge SSI. The primary objective of this study was to determine trends in admissions for post-discharge SSI at a South African quaternary/teaching hospital. The secondary objective was to determine trends in mortality rates for these admissions.

Methods: This was a retrospective review of adult admissions for post-discharge SSI at a quaternary/teaching South African hospital between 2006 and 2015. Admissions for post-discharge SSI were identified using the hospital administrative database and appropriate International Classification of Disease, $10^{\text {th }}$ Revision codes. Mortality was determined from the discharge disposition for each admission. Data were analysed with simple regression and trend line statistics. The geospatial distribution of post-discharge SSI, based on the residential postal codes recorded on the hospital administrative database for each admission, was determined using the Power Map® software program.

Results: There was no change in admissions for post-discharge SSI over the study period $(p=0.17)$. Mortality in elderly admissions declined during the study period $(\mathrm{p}=0.03)$. Most admissions for post-discharge SSIs originated from urban areas.

Conclusion: Despite the implementation of universal SSI prevention methods, admissions for post-discharge SSI remained consistent during the study period. Urban areas appeared to be more severely affected by post-discharge SSI than rural areas. Additional prevention methods for post-discharge SSI are required.

Keywords: surgical site infection, post-discharge, admissions, mortality, South Africa
\end{abstract}

S Afr J Surg 2019;57(4)

http://dx.doi.org/10.17159/2078-5151/2019/v57n4a2915

\section{Introduction}

Surgical site infection (SSI) is an important postoperative complication, ${ }^{1,2}$ and contributes towards increased healthcare expenditure and resource utilisation at healthcare facilities. ${ }^{3}$ Postoperative surgical site infection can occur as an inhospital event or a post-discharge event. ${ }^{4,5}$ The incidence of inpatient and post-discharge SSI differs according to surgical procedure. $^{4}$

Inpatient data from the African Surgical Outcomes Study (ASOS) has highlighted the importance of SSI in African settings. ${ }^{6}$ However, loss to follow up once the patient is discharged from hospital is a challenge, ${ }^{7}$ and most studies from African settings are usually investigations of inpatient outcomes only. Even ASOS did not extend investigations of most postoperative complications beyond hospital discharge. ${ }^{6}$ There is a gap in the literature regarding post-discharge SSI in an African setting. Addressing this deficiency could be important for three reasons. Firstly, it could assist public health specialists and surgeons in deciding how resources should be allocated within healthcare facilities for SSI-related admissions. Secondly, it could assist with the development of interventions aimed at reducing post-discharge SSI. Lastly, the demand for surgical procedures is increasing on the African continent, ${ }^{8}$ and some of these surgical cases are at risk for SSI. ${ }^{6}$

The primary objective of this study was to determine trends in admissions for post-discharge SSI at a South African quaternary/teaching hospital. The secondary objective of this study was to determine trends in mortality for these admissions.

\section{Methods}

\section{Study design and setting}

This study was a retrospective review of data obtained from the admissions database of the Inkosi Albert Luthuli Central 
Hospital (IALCH), located in Durban, South Africa. This quaternary/teaching hospital has 850 beds and offers various specialised medical and surgical services to the populace of the KwaZulu-Natal province. A description of surgical procedure rates at IALCH is provided in Table 1. Cardiac surgery procedures were defined as procedures performed on the heart by cardiac surgeons. Noncardiac surgery procedures were defined as procedures which did not meet the definition of cardiac surgery, and are stratified by surgical sub-specialty (Table 1).

\section{Study sample}

The study sample was comprised of all adult admissions at IALCH between 01 January 2006 and 31 December 2015, with a primary International Classification of Disease $10^{\text {th }}$ Revision (ICD-10) diagnosis indicative of SSI. The ICD10 diagnosis codes used to identify admissions with postdischarge SSI are shown in Table 2. In addition, ICD-10 codes were broadly classified as SSIs not involving grafts/prostheses (ICD-10 code T81.4) and SSIs involving grafts/prostheses (all remaining ICD-10 codes listed in Table 2).

\section{Data source and data description}

The data for this study were extracted directly from the hospital admissions database and stored as a Microsoft Excel ${ }^{\circledR}$ file in preparation for statistical analysis. Beside the ICD-10 primary diagnosis code for SSI, variables contained in the database included: date of admission, admission age and gender, discharge disposition, and residential postal code. Mortality was determined by reviewing the discharge disposition recorded for each admission in the hospital admissions database.

\section{Data analysis}

Characteristics of the entire study sample were analysed using descriptive statistical methods and are presented as frequencies and percentages, or rates with confidence intervals (CI). Simple regression and trend line analysis was used to investigate trends in post-discharge SSI admissions and mortality in these admissions. Trends analyses were stratified according to age, gender, the nature of SSI ICD10 code, and broad surgical category (i.e. noncardiac surgery versus cardiac surgery). The direction of a trend was determined from the slope of the trend line, with a negative slope indicating a declining trend while a positive slope would be indicative of an increasing trend. The $\mathrm{R}^{2}$ value from the simple regression analysis was used to interpret the strength of a trend. Trends with an $\mathrm{R}^{2}$ value of $<0.5000$ were considered "weak", trends with an $\mathrm{R}^{2}$ value of $0.5000-0.7000$ were considered "moderate", and trends with an $\mathrm{R}^{2}$ value of $>0.7000$ were considered "strong". The descriptive statistics and simple regression/trend line analyses were performed using Microsoft Excel®. For the trends analysis, a p-value $<0.05$ was considered a statistically significant result.

The geospatial distribution of post-discharge SSI admissions was semi-quantitatively determined using the Power Map ${ }^{\circledR}$ add-on software for Microsoft Excel ${ }^{\circledR}$. Briefly, the Power Map ${ }^{\circledR}$ add-on software uses postal codes

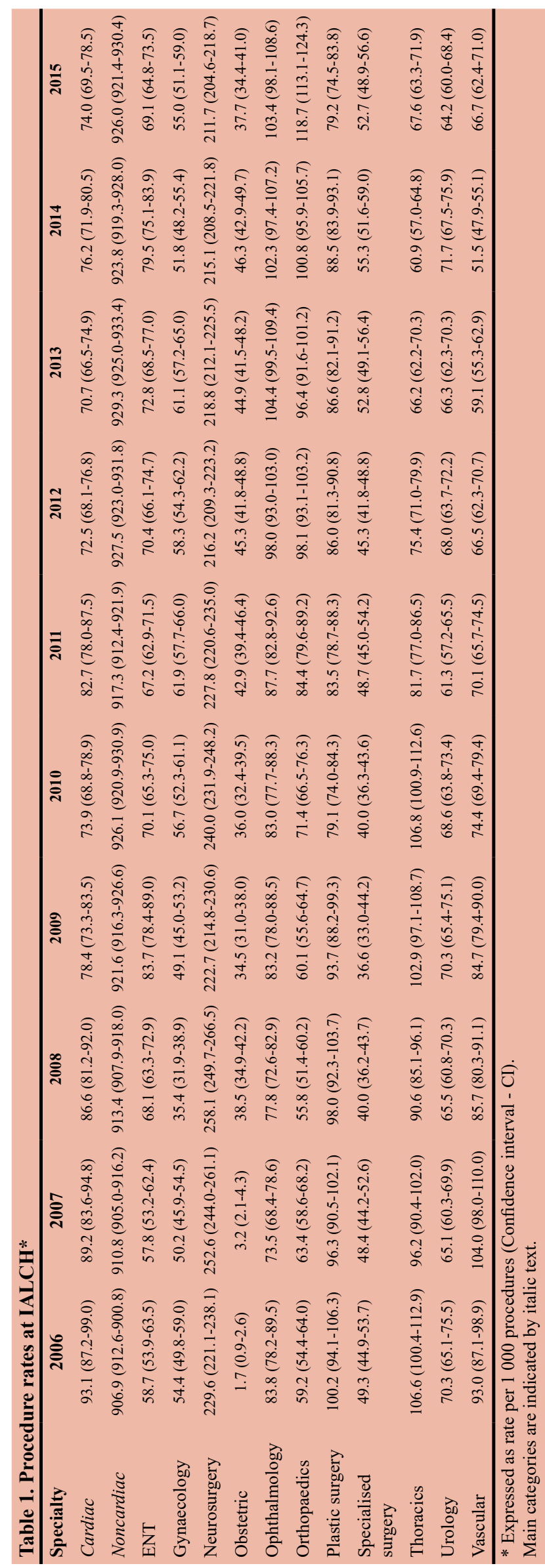


Table 2. ICD-10 diagnosis codes used to identify admissions with a post-discharge SSI

\begin{tabular}{|c|c|}
\hline Code & Description \\
\hline T81.4 & Infection following a procedure, not elsewhere classified \\
\hline $\mathrm{T} 85.7$ & $\begin{array}{l}\text { Infection and inflammatory reaction due to other internal } \\
\text { prosthetic devices, implants and grafts }\end{array}$ \\
\hline T82.6 & $\begin{array}{l}\text { Infection and inflammatory reaction due to cardiac valve } \\
\text { prosthesis }\end{array}$ \\
\hline T82.7 & $\begin{array}{l}\text { Infection and inflammatory reaction due to other cardiac } \\
\text { and vascular devices, implants and grafts }\end{array}$ \\
\hline T83.5 & $\begin{array}{l}\text { Infection and inflammatory reaction due to prosthetic } \\
\text { device, implant and graft in urinary system }\end{array}$ \\
\hline T83.6 & $\begin{array}{l}\text { Infection and inflammatory reaction due to implanted } \\
\text { penile prosthesis }\end{array}$ \\
\hline T84.5 & $\begin{array}{l}\text { Infection and inflammatory reaction due to internal joint } \\
\text { prosthesis }\end{array}$ \\
\hline T84.6 & $\begin{array}{l}\text { Infection and inflammatory reaction due to internal } \\
\text { fixation device }\end{array}$ \\
\hline T84.7 & $\begin{array}{l}\text { Infection and inflammatory reaction due to other internal } \\
\text { orthopaedic prosthetic devices, implants and grafts }\end{array}$ \\
\hline
\end{tabular}

in the Microsoft Excel ${ }^{\circledR}$ database and blank maps available through Microsoft Bing ${ }^{\circledR}$ to create new maps which display the geospatial distribution of a given characteristic, which in this instance would be admissions for post-discharge SSI. The display options for the map can be set such that areas with a high density of admissions for post-discharge SSI appear as red "hot spots", while areas with a low density of admissions for post-discharge SSI would appear green. Areas with an intermediate density of admissions for post-discharge SSI would appear yellow.

\section{Results}

The study sample consisted of 1240 post-discharge SSI admissions which were recorded during the 10-year study period. The mean age of the study sample was 46.9 years with $15.3 \%$ (190 admissions) of the study sample aged $>65$ years. Six hundred and sixty eight $(53.8 \%)$ admissions in the study sample were male. A total of $808(67.1 \%)$ admissions did not involve SSI of grafts/prostheses. Mortality across the study period was $9.5 \%$ (118 admissions).

The results of the trends analysis are shown in Figures 1 and 2. A weak, but statistically significant trend toward a reduction in mortality amongst elderly admissions with postdischarge SSI $\left(\mathrm{R}^{2}=0.4847, \mathrm{p}=0.03\right)$ was observed (Figure $2)$. There were no other statistically significant trends for the admission and mortality outcomes investigated in this study.

The geospatial distribution of SSI admissions in this study is shown in Figure 3. A high-density area of post-discharge SSI admissions was noted around Durban, as well as several periurban areas surrounding Durban. Post-discharge admissions for SSI from rural areas in the north and south, as well as the midlands of KwaZulu-Natal province were less common.

\section{Discussion}

Along with the inpatient findings reported in ASOS, ${ }^{6}$ this study contributes toward a better overall understanding of SSI on the African continent. Most admissions in the study sample were of younger age. There is evidence to suggest that the risk of SSI increases up to the age of 65 years of age, following which there is a decrease in risk. ${ }^{9}$ In addition, most postdischarge SSIs were in admissions that did not have recent
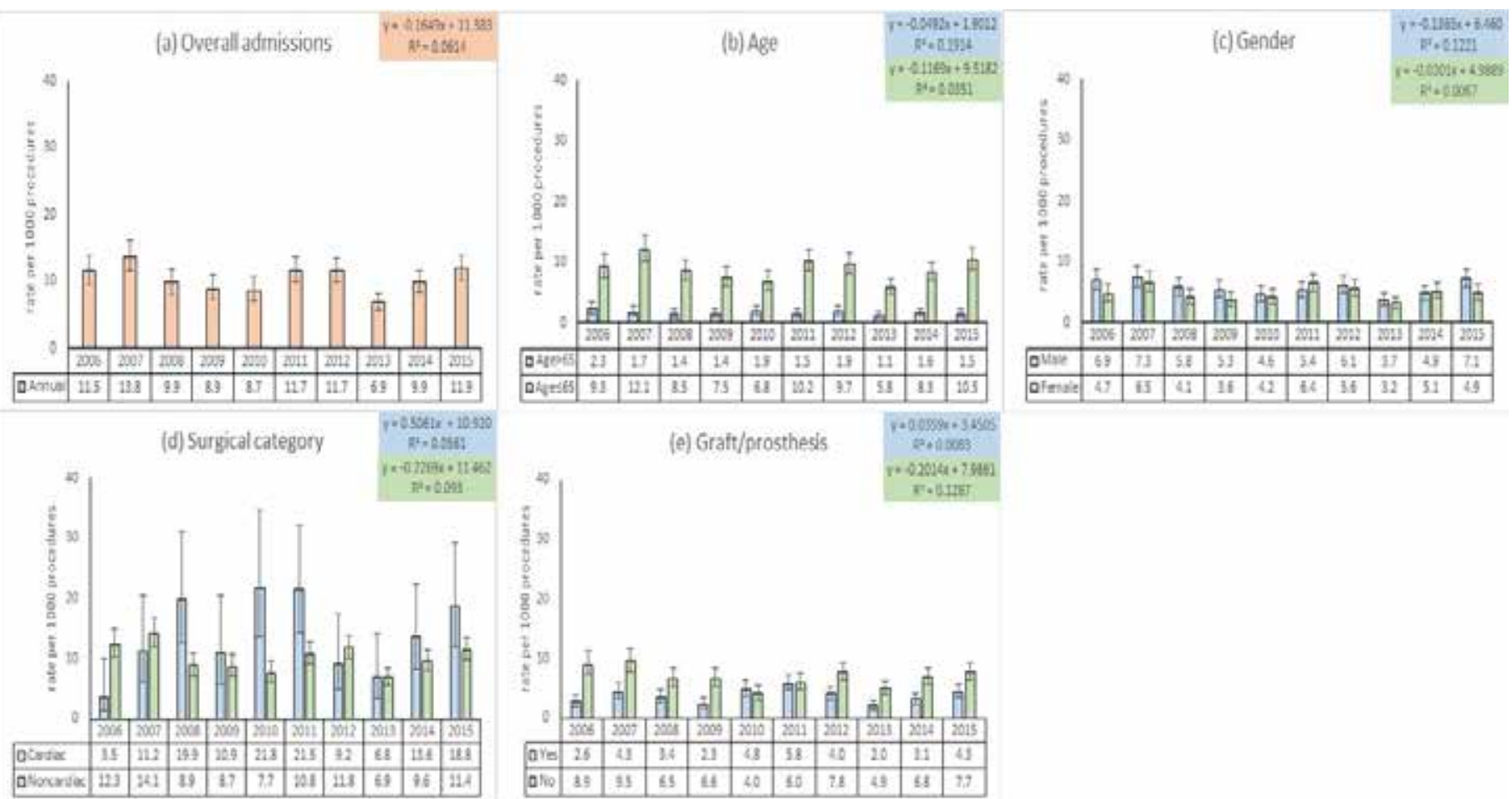

Figure 1. Overall and stratified trends in admissions for post-discharge SSI*

* Colour-coded boxes contain trend line equations and $\mathrm{R}^{2}$ values for corresponding colour-coded variables and sub-categories.

Error bars on graph indicate confidence intervals for estimates. 

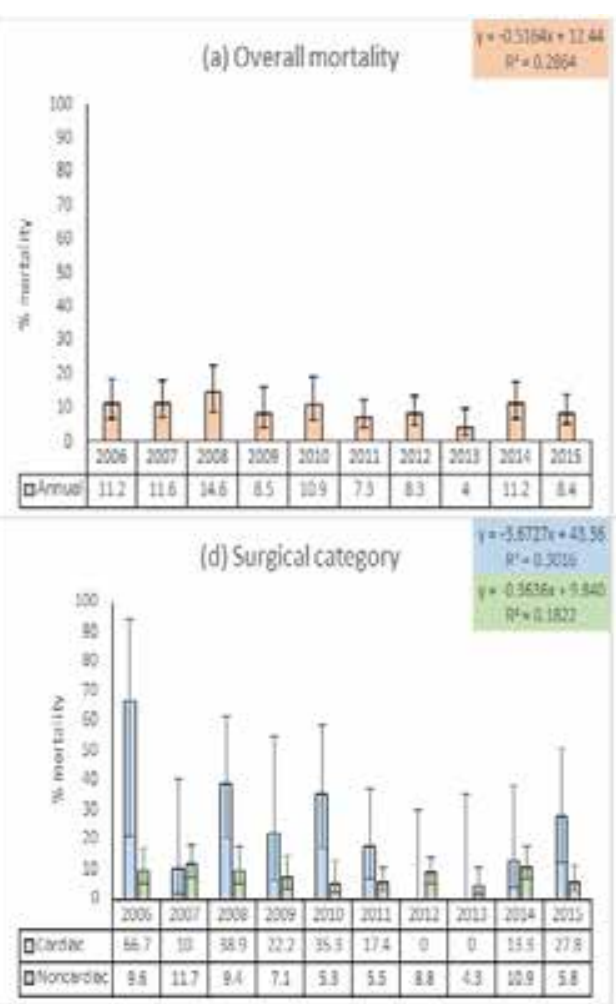
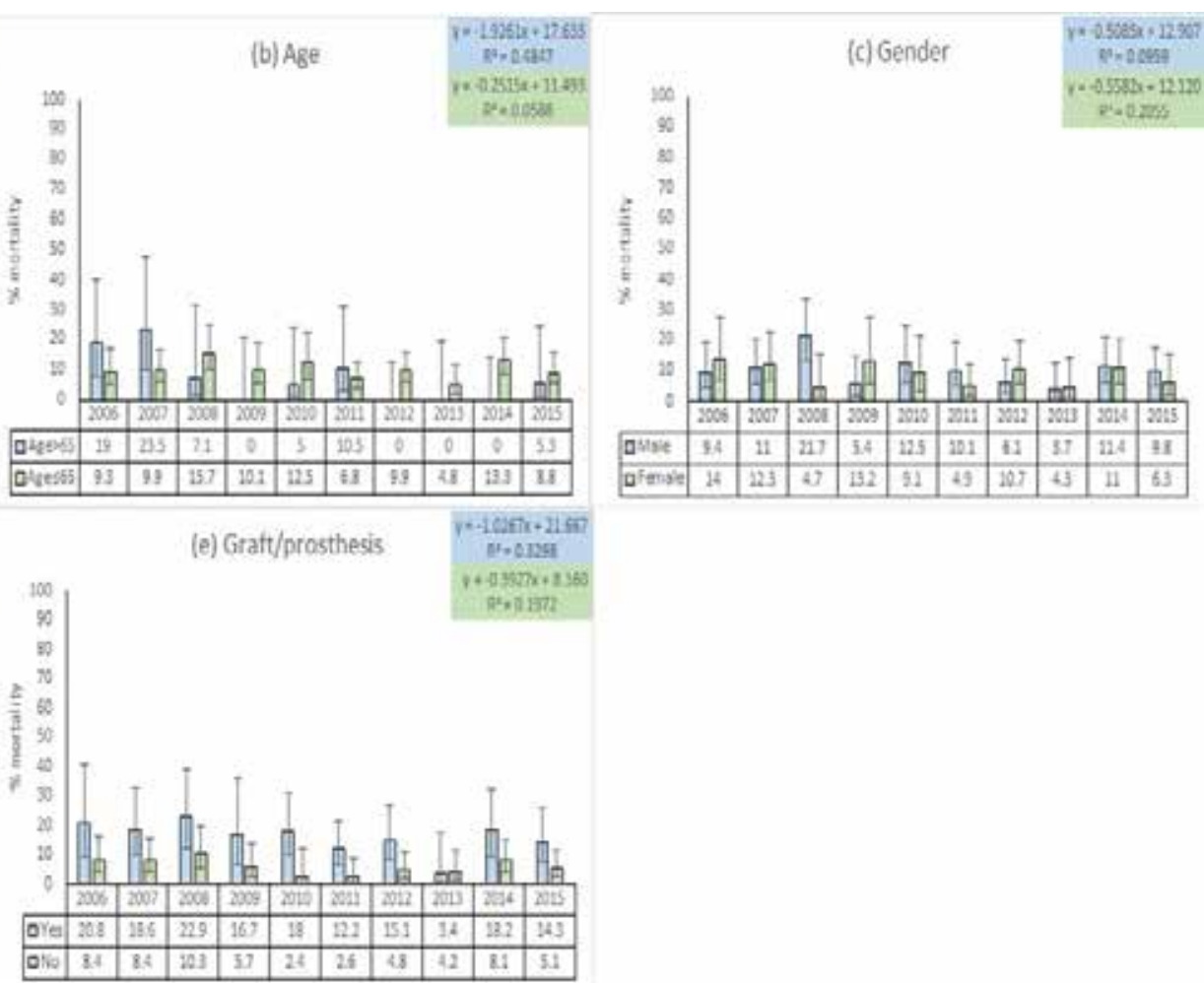

Figure 2. Overall and stratified trends for mortality in admissions with post-discharge SSI

* Colour-coded boxes contain trend line equations and $\mathrm{R}^{2}$ values for corresponding colour-coded variables and sub-categories. Error bars on graph indicate confidence intervals for estimates.

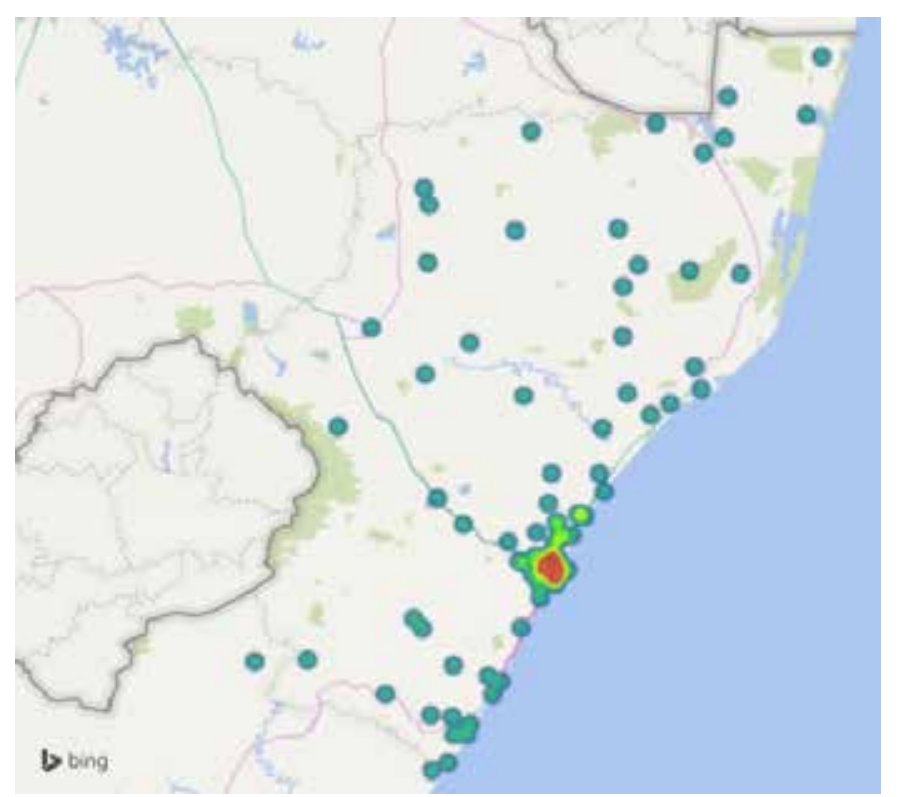

Figure 3. Geospatial distribution of post-discharge admissions for SSI in this study*

* Across entire study period (2006-2015). With reference to the density of post-discharge admissions for SSI: Green - area with low density, yellow area with intermediate density, and red - area with high density.

graft/prosthesis procedures. Surgical procedures involving grafts or prostheses are more likely in older patients rather than younger patients, which might explain this finding. For example, while there has been an increase in knee arthroplasty amongst persons younger than 60 years of age, the majority of arthroplasties continue to be performed in persons older than 60 years. ${ }^{10}$

The findings of the trends analysis suggest admissions for post-discharge SSI at IALCH, irrespective of stratification level, have remained consistent during the 10-year study period. Ideally, there should have been a declining trend in postdischarge SSI during the study period. The hospital follows the World Health Organization (WHO) recommendations for the prevention of SSI, ${ }^{11}$ and no new policies were specifically implemented during the study period. This demonstrates that although WHO recommendations for SSI prevention have been adopted at IALCH, further efforts are required to significantly reduce admissions for post-discharge SSI. Surgical site infection is multifactorial. Although the WHO recommendations seek to prevent SSI by addressing risk factors at the facility and healthcare worker levels, addressing risk factors at the patient level is also important. A potential method of reducing post-discharge SSI is patient empowerment through health promotion and educational initiatives. These health promotion activities and educational materials should be related to risk factor avoidance and proper wound care following discharge from hospital. ${ }^{12}$ Health promotion materials would need to be culturally relevant to African settings to be effective. Early detection and treatment of post-discharge SSI might reduce the chances of the SSI advancing to the point where it requires patient hospitalisation 
for treatment. Mobile phone technology has been used in the surveillance of post-discharge SSI in some settings. ${ }^{13,14}$ This intervention has the potential to identify post-discharge SSI at an early stage. Patients can then receive timeous treatment. As access to mobile phones in African settings is increasing, ${ }^{15}$ post-discharge SSI surveillance through a mobile phone-based intervention in these settings should be considered.

One in ten admissions for post-discharge SSI in this study died in hospital. Overall mortality in patients with SSI in ASOS was $9.0 \%$, but ranged between $5.2 \%$ and $22.4 \%$ depending on the extent of the SSI. ${ }^{6}$ However, ASOS was a study of inpatient outcomes and did not investigate postdischarge complications. ${ }^{6}$ As the mortality findings reported for this study of post-discharge SSI admissions are similar to those reported for inpatient SSI in ASOS, ${ }^{6}$ it would appear that inpatient and post-discharge SSIs have a similar importance with regard to mortality in African settings. This once again highlights the importance of prevention of preand post-discharge SSI, as well as the timely diagnosis and treatment of SSI in African settings. Another finding of this study was a trend toward a reduction in mortality amongst elderly admissions with post-discharge SSI. However, we do believe that this finding is artefactual, and is likely explained by factors which lie beyond the scope of the dataset used in this study.

Rural patient groups have been reported as having worse postoperative outcomes (including SSI) when compared with their urban counterparts. ${ }^{16}$ While our semi-quantitative geospatial analysis revealed high density clusters of postdischarge admissions in Durban, this finding should be interpreted with caution as it may have been influenced by our dichotomous definition of population density (whether clusters of admissions were located around a major urban centre or not), as well as the inability of the semi-quantitative analysis to account for socioeconomics, demographic group, or other potential confounders.

Strengths of this study include the large sample size and the ten-year study duration, which allowed for an appropriate trends analysis to be conducted. Another strength of this study was that the geospatial distribution of post-discharge admissions for SSI was mapped in KwaZulu-Natal. This study also had several limitations. The data used in this study were from a single, quaternary-level South African hospital. Therefore, the findings might not necessarily be generalisable to other healthcare facilities in South Africa or other African settings. There is also a possibility that some patients, such as those patients from rural areas, may have been admitted or managed for post-discharge SSI at another healthcare facility much closer to their place of residence. These would represent "missed" post-discharge SSIs. There might have also been some admissions which were incorrectly coded on the hospital administrative database as having SSI. Conversely, there might have been some admissions with a primary diagnosis of SSI which were missed. The medical informatics system at IALCH has been changed several times between 2006 and 2015, during which some of the finer details related to procedures performed at the hospital were lost. Therefore, surgical procedures have been broadly classified in this study as cardiac or noncardiac (with sub-specialties) procedures. Data related to other comorbidities and medication use were not recorded on the hospital administrative database, and therefore could not be investigated in this study. The specific cause of death could not be established for those patients who died in hospital. Lastly, there was no sub-classification of SSI according to extent (superficial, deep incisional, or organ space) in this study.

\section{Conclusion}

Despite implementation of universal SSI prevention methods, the number of admissions for post-discharge SSI at IALCH remained consistent during the study period. Additional efforts are required to reduce the number of post-discharge SSI admissions at IALCH. Such efforts would need to consider the multifactorial aetiology of SSI. A prevention package which simultaneously addresses risk factors at various levels would be best suited for reducing SSI in this setting. Patients from urban areas appear to be more affected by post-discharge SSI than patients from rural areas. Further research, which accounts for socioeconomic and demographic characteristics, is required to confirm this finding. While this study had strengths, it also had limitations which should be addressed in future studies on the topic.

\section{Ethics approval}

This study was a component of a larger healthcare utilisation project which was approved by the Biomedical Research Ethics Committee of the University of KwaZulu-Natal, the KwaZulu-Natal Provincial Department of Health (Protocol number: BE595/16), and the Medical Manager of IALCH.

\section{Conflicts of interest/Commercial interests}

None.

\section{Orchid}

TE Madiba http://orcid.org/0000-0002-0155-9143

Y Moodley http://orcid.org/0000-0002-0957-6482

\section{REFERENCES}

1. Ban KA, Minei JP, Laronga $\mathrm{C}$, et al. American College of Surgeons and Surgical Infection Society: Surgical Site Infection Guidelines, 2016 Update. J Am Coll Surg. 2017;224(1):59-74. Available from: http://dx.doi.org/10.1016/j. jamcollsurg.2016.10.029 PMID: 27915053

2. GlobalSurg-Collaborative. Surgical site infection after gastrointestinal surgery in high-income, middle-income, and low-income countries: a prospective, international, multicentre cohort study. Lancet Infect Dis. 2018;18(5):516-25. Available from: http://dx.doi.org/10.1016/s1473-3099(18)30101-4 PMID: 29452941

3. Shepard J, Ward W, Milstone A, et al. Financial impact of surgical site infections on hospitals: the hospital management perspective. JAMA Surg. 2013;148(10):907-14. Available from: http://dx.doi.org/10.1001/jamasurg.2013.2246 PMID: 23965750 
4. Sands K, Vineyard G, Platt R. Surgical site infections occurring after hospital discharge. J Infect Dis. 1996;173(4):963-70. PMID: 8603978

5. Woelber E, Schrick EJ, Gessner BD, Evans HL. Proportion of Surgical Site Infections Occurring after Hospital Discharge: A Systematic Review. Surg Infect (Larchmt). 2016;17(5):510-9. Available from: http://dx.doi.org/10.1089/sur.2015.241 PMID: 27463235

6. Biccard BM, Madiba TE, Kluyts HL, et al. Perioperative patient outcomes in the African Surgical Outcomes Study: a 7-day prospective observational cohort study. Lancet. 2018;391(10130):1589-98. Available from: http://dx.doi. org/10.1016/s0140-6736(18)30001-1 PMID: 29306587

7. Abbas M, Pittet D. Surgical site infection prevention: a global priority. J Hosp Infect. 2016;93(4):319-22. Available from: http://dx.doi.org/10.1016/j.jhin.2016.06.002 PMID: 27388057

8. Musa J. Generating evidence for surgical practice in Africa: the role of clinical research. Afr J Paediatr Surg. 2012;9(1):62-5. Available from: http://dx.doi.org/10.4103/0189-6725.93310 PMID: 22382107

9. Kaye KS, Schmader KE, Sawyer R. Surgical site infection in the elderly population. Clin Infect Dis. 2004;39(12):183541. Available from: http://dx.doi.org/10.1086/425744 PMID: 15578408

10. Losina E, Katz JN. Total knee arthroplasty on the rise in younger patients: are we sure that past performance will guarantee future success? Arthritis Rheum. 2012;64(2):339-41. Available from: http://dx.doi.org/10.1002/art.33371 PMID: 22252374

11. Allegranzi B, Zayed B, Bischoff P, et al. New WHO recommendations on intraoperative and postoperative measures for surgical site infection prevention: an evidence-based global perspective. Lancet Infect Dis. 2016;16(12):e288-e303. Available from: http://dx.doi.org/10.1016/s14733099(16)30402-9 PMID: 27816414

12. Tartari E, Weterings V, Gastmeier P, et al. Patient engagement with surgical site infection prevention: an expert panel perspective. Antimicrob Resist Infect Control. 2017;6:45 Available from: http://dx.doi.org/10.1186/s13756-017-0202-3 PMID: 28507731

13. Castillo E, McIsaac C, MacDougall B, Wilson D, Kohr R. PostCaesarean Section Surgical Site Infection Surveillance Using an Online Database and Mobile Phone Technology. J Obstet Gynaecol Can. 2017;39(8):645-51.e641. Available from: http:// dx.doi.org/10.1016/j.jogc.2016.12.037 PMID: 28729097

14. Pathak A, Sharma S, Sharma M, Mahadik VK, Lundborg CS. Feasibility of a Mobile Phone-Based Surveillance for Surgical Site Infections in Rural India. Telemed J E Health. 2015;21(11):946-9. Available from: http://dx.doi.org/10.1089/ tmj.2014.0199 PMID: 25748641

15. Aker JC, Mbiti IM. Mobile phones and economic development in Africa. J Econ Perspect. 2010;24(3):207-32. PMID: N/A

16. Kong VY, Van der Linde S, Aldous C, Handley JJ, Clarke DL. Quantifying the disparity in outcome between urban and rural patients with acute appendicitis in South Africa. S Afr Med J. 2013;103(10):742-5. Journal of Economic Perspectives http:// dx.doi.org/10.7196/SAMJ.7109 PMID: 24079626 